\title{
Kinetics of viral load and antibody response in relation to COVID-19 severity
}

\author{
Yanqun Wang, ${ }^{1}$ Lu Zhang, ${ }^{2}$ Ling Sang, ${ }^{1}$ Feng Ye, ${ }^{1}$ Shicong Ruan, ${ }^{3}$ Bei Zhong, ${ }^{4}$ Tie Song, ${ }^{5}$ Abeer N. Alshukairi, ${ }^{6}$ Rongchang Chen, ${ }^{7}$ \\ Zhaoyong Zhang, ${ }^{1}$ Mian Gan, ${ }^{1}$ Airu Zhu, ${ }^{1}$ Yongbo Huang, ${ }^{1}$ Ling Luo, ${ }^{1}$ Chris Ka Pun Mok, ${ }^{8}$ Manal M. Al Gethamy, ${ }^{9}$ Haitao Tan, ${ }^{3}$ \\ Zhengtu Li, ${ }^{1}$ Xiaofang Huang, ${ }^{1}$ Fang Li, ${ }^{1}$ Jing Sun, ${ }^{1}$ Yanjun Zhang, ${ }^{1}$ Liyan Wen, ${ }^{1}$ Yuming Li, ${ }^{1}$ Zhao Chen, ${ }^{1}$ Zhen Zhuang, ${ }^{1}$ \\ Jianfen Zhuo, ${ }^{1}$ Chunke Chen, ${ }^{1}$ Lijun Kuang, ${ }^{1}$ Junxiang Wang, ${ }^{1}$ Huibin Lv, ${ }^{8}$ Yongliang Jiang, ${ }^{4}$ Min Li, ${ }^{4}$ Yimin Lin, ${ }^{4}$ Ying Deng, ${ }^{4}$ \\ Lan Tang, ${ }^{4}$ Jieling Liang, ${ }^{4}$ Jicheng Huang, ${ }^{10}$ Stanley PerIman,, ${ }^{11}$ Nanshan Zhong, Jingxian Zhao, ${ }^{1}$ J.S. Malik Peiris, ${ }^{8}$ \\ Yimin $\mathrm{Li},{ }^{1}$ and Jincun Zhao, ${ }^{1,2}$ \\ 'State Key Laboratory of Respiratory Disease, National Clinical Research Center for Respiratory Disease, Guangzhou Institute of Respiratory Health, First Affiliated Hospital, and Institute of Infectious

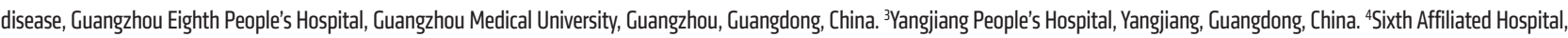 \\ Guangzhou Medical University, Qingyuan People's Hospital, Qingyuan, Guangdong, China. ${ }^{5}$ Guangdong Provincial Center for Disease Control and Prevention, Guangzhou, Guangdong, China. ${ }^{6}$ King Faisal \\ Specialist Hospital and Research Centre, Jeddah, Saudi Arabia. 'Shenzhen Institute of Respiratory Disease, First Affiliated Hospital (Shenzhen People's Hospital), South University of Science and Technology \\ of China, Shenzhen, Guangdong, China. ${ }^{8} \mathrm{HKU}$-Pasteur Research Pole, School of Public Health, Li Ka Shing Faculty of Medicine, University of Hong Kong, Hong Kong, China. ${ }^{9} \mathrm{Al}$ Nour Specialist Hospital, \\ Makkah, Saudi Arabia. ${ }^{10}$ Technology Centre, Guangzhou Customs, Guangzhou, Guangdong, China. "Department of Microbiology and Immunology, University of lowa, lowa City, lowa, USA.
}

\begin{abstract}
Severe acute respiratory syndrome coronavirus 2 (SARS-CoV-2) is the causative agent for coronavirus 2019 (COVID-19) pneumonia. Little is known about the kinetics, tissue distribution, cross-reactivity, and neutralization antibody response in patients with COVID-19. Two groups of patients with RT-PCR-confirmed COVID-19 were enrolled in this study: 12 severely ill patients in intensive care units who needed mechanical ventilation and 11 mildly ill patients in isolation wards. Serial clinical samples were collected for laboratory detection. Results showed that most of the severely ill patients had viral shedding in a variety of tissues for $20-40$ days after onset of disease (8/12, 66.7\%), while the majority of mildly ill patients had viral shedding restricted to the respiratory tract and had no detectable virus RNA 10 days after onset $(9 / 11,81.8 \%$ ). Mildly ill patients showed significantly lower IgM response compared with that of the severe group. IgC responses were detected in most patients in both the severe and mild groups at 9 days after onset, and remained at a high level throughout the study. Antibodies cross-reactive to SARS-CoV and SARS-CoV-2 were detected in patients with COVID-19 but not in patients with MERS. High levels of neutralizing antibodies were induced after about 10 days after onset in both severely and mildly ill patients which were higher in the severe group. SARS-CoV-2 pseudotype neutralization test and focus reduction neutralization test with authentic virus showed consistent results. Sera from patients with COVID-19 inhibited SARS-CoV-2 entry. Sera from convalescent patients with SARS or Middle East respiratory syndrome (MERS) did not. Anti-SARS-CoV-2 $\mathrm{S}$ and $\mathrm{N}$ IgG levels exhibited a moderate correlation with neutralization titers in patients' plasma. This study improves our understanding of immune response in humans after SARS-CoV-2 infection.
\end{abstract}

\section{Introduction}

Before November 2019, 6 coronaviruses (CoVs) were known to infect humans and cause respiratory disease: OC43, 229E, HKU1, and NL63, 4 community-acquired CoVs that are endemic in humans (1-4), and severe acute respiratory syndrome CoV (SARS$\mathrm{CoV})(5)$ and Middle East respiratory syndrome CoV (MERS-CoV)

\section{Related Commentary: p. 5112}

Authorship note: YW, LZ, LS, FY, SR, BZ, TS, ANA, and RC contributed equally to this work.

Conflict of interest: The authors have declared that no conflict of interest exists. Copyright: () 2020, American Society for Clinical Investigation.

Submitted: April 2, 2020; Accepted: June 17, 2020; Published: August 31, 2020.

Reference information: J Clin Invest. 2020;130(10):5235-5244.

https://doi.org/10.1172/JCl138759.
(6), 2 highly pathogenic CoVs that have zoonotic transmission followed by variable transmission between humans. Recently, a novel coronavirus first identified in Wuhan, Hubei province, China, in late 2019, has spread worldwide to cause a pandemic of pneumonia (7). The novel CoV was named SARS-CoV-2 and the disease was called coronavirus disease 2019 (COVID-19) (8). Robust human-to-human transmission of SARS-CoV-2 led to a pandemic involving every province of China and then more than 203 countries and territories (9). As of August 21, 2020, over 22 million cases of COVID-19 have been reported to the World Health Organization (WHO), including 780,000 deaths (10).

SARS-CoV-2 belongs to the lineage B betacoronavirus and has high nucleotide homology with bat SARS-like CoV and SARS-CoV (11). SARS-CoV-2 causes severe respiratory illness similar to SARSCoV. Older adults and individuals with comorbidities are at higher risk for severe disease (12-14). Little is known about the immune 
Table 1. Demographics and clinical outcomes of patients infected with SARS-CoV-2

\begin{tabular}{|c|c|c|c|c|c|c|c|c|}
\hline & $\begin{array}{l}\text { Patient } \\
\text { number }\end{array}$ & Sex & $\begin{array}{l}\text { Age, } \\
\text { years }\end{array}$ & City, Country & $\begin{array}{l}\text { Visited Wuhan } \\
\text { Hubei, China }\end{array}$ & $\begin{array}{l}\text { Direct contact with } \\
\text { confirmed cases }\end{array}$ & $\begin{array}{l}\text { Receiving mechanical } \\
\text { ventilation }\end{array}$ & $\begin{array}{l}\text { Clinical } \\
\text { outcome }\end{array}$ \\
\hline \multirow{9}{*}{ Group A: severely ill patients $(n=12)$} & PT2 & M & 49 & Guangzhou, China & Y & $Y$ & Y & Still in ICU \\
\hline & PT4 & M & 53 & Guangzhou, China & $\mathrm{N}$ & Y & Y & Still in ICU \\
\hline & PT5 & M & 61 & Guangzhou, China & Y & $\mathrm{N}$ & Y & Still in ICU \\
\hline & PT6 & M & 42 & Guangzhou, China & Y & NA & Y & Transferred out of ICU \\
\hline & PT8 & M & 58 & Guangzhou, China & $\mathrm{N}$ & Y & Y & Still in ICU \\
\hline & PT9 & M & 42 & Guangzhou, China & Y & Y & Y & Transferred out of ICU \\
\hline & P10 & M & 79 & Guangzhou, China & $\mathrm{N}$ & Y & Y & Still in ICU \\
\hline & PT11 & M & 61 & Yangjiang, China & Y & Y & Y & Still in ICU \\
\hline & PT12 & $\mathrm{F}$ & 60 & Yangjiang, China & Y & Y & Y & Still in ICU \\
\hline \multirow{7}{*}{ Group B: mildly ill patients $(n=11)$} & PT17 & M & 24 & Yangjiang, China & Y & $\mathrm{N}$ & $\mathrm{N}$ & Discharged \\
\hline & PT18 & M & 35 & Yangjiang, China & Y & Y & $\mathrm{N}$ & Discharged \\
\hline & PT19 & M & 69 & Yangjiang, China & Y & $\mathrm{N}$ & $\mathrm{N}$ & Discharged \\
\hline & PT20 & M & 77 & Yangjiang, China & Y & Y & $\mathrm{N}$ & Discharged \\
\hline & PT21 & M & 52 & Qingyuan, China & Y & NA & $\mathrm{N}$ & Discharged \\
\hline & PT22 & M & 50 & Qingyuan, China & Y & NA & $\mathrm{N}$ & Discharged \\
\hline & PT23 & M & 65 & Qingyuan, China & Y & NA & $\mathrm{N}$ & Isolation ward \\
\hline
\end{tabular}

NA indicates not available.

response and its relationship with clinical outcomes in patients infected with SARS-CoV-2. Information about the kinetics of virus replication, neutralizing antibody responses, and cross-reactivity with other human respiratory CoVs are also required for diagnosis, prognosis, and epidemiology investigations.

We found, by monitoring different tissues, that COVID-19 patients with various severities of disease showed different patterns of viral shedding and antibody responses. Severely ill patients had more prolonged viral shedding in a variety of tissues than mildly ill patients. IgM responses in mildly ill patients were much lower than those observed in severely ill patients, indicating that IgM detection in mildly ill patients was not sensitive and efficient. SARS-CoV-2-specific antibodies were found in tissues outside the respiratory tract in severely ill patients. Detection of antibody responses in urine and other body fluids could be used as a marker to determine disease severity. By using plasma from patients with SARS, MERS, or COVID-19, strong cross-reactivities were detected between SARS-CoV-2 and SARS-CoV, but not MERS-CoV, which is important information for differential diagnosis in Middle East countries. Antibodies against $\mathrm{N}$ or S protein were correlated with neutralizing antibody titers, which may be useful when screening convalescent plasma for passive transfusion therapy.

Results

Patients and clinical information. Twenty-three patients with laboratory-confirmed SARS-CoV-2 were enrolled in this study. Serial clinical samples were collected every $3-4$ days for profiling the kinetics of antibody responses and viral loads during SARS-CoV-2 infection. A total of 12 severely ill patients and 11 mildly ill patients from 3 hospitals were enrolled in this study (Table 1). Most patients were older than 50 years with a median age of 56 years (24-82 years). At the time of writing, most mildly ill patients $(8 / 11$, $72.7 \%$ ) were discharged from the hospital, whereas the majority of severely ill patients $(10 / 12,83.3 \%)$ were still in the intensive care unit (ICU). Most of the patients enrolled in the study had visited Wuhan or had direct contact with other confirmed cases.

Prolonged viral shedding from multiple sites in severely patients. A total of 461 clinical samples (84 nasal swabs, 59 throat swabs, 36 sputum samples, 90 fecal samples, 79 urine samples, 113 plasma samples) and 1 biopsy of gastric juice were obtained from 23 severely or mildly ill patients. Most patients with severe disease had viral shedding for up to 30-40 days after onset, and a majority of mildly ill patients had no detectable viral loads 15 days after onset (Figure 1). There was a significant difference in the peak viral load $(P<0.001, t$ test) between severe and mild cases. The viral loads in respiratory samples were higher in the severe group than in the mild group, and gradually declined over time. SARS-CoV-2 virus was mainly detected in respiratory samples (nasal swabs, throat swabs, and sputum) (Figure 1, A-C). However, in most of the severely ill patients, feces remained positive for viral RNA over a prolonged time (Figure 1D). Periodically, urine and plasma also tested positive (Figure 1, E and F).

Mildly ill patients have lower IgM responses against SARS-CoV-2 than severely ill patients. To understand the kinetics of the antibody 
A

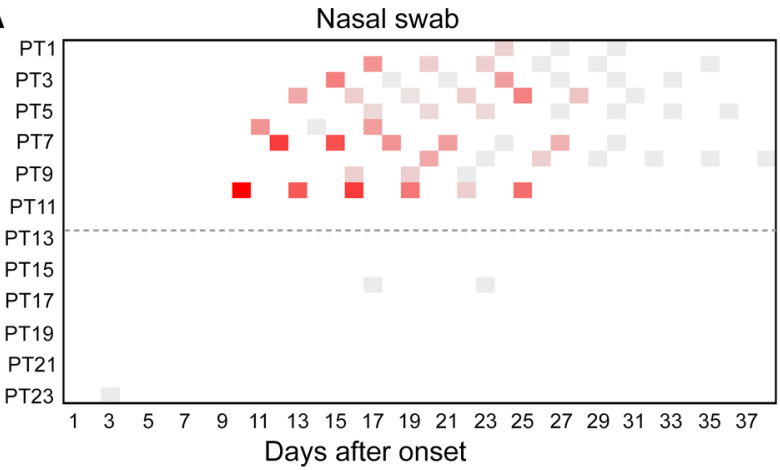

B

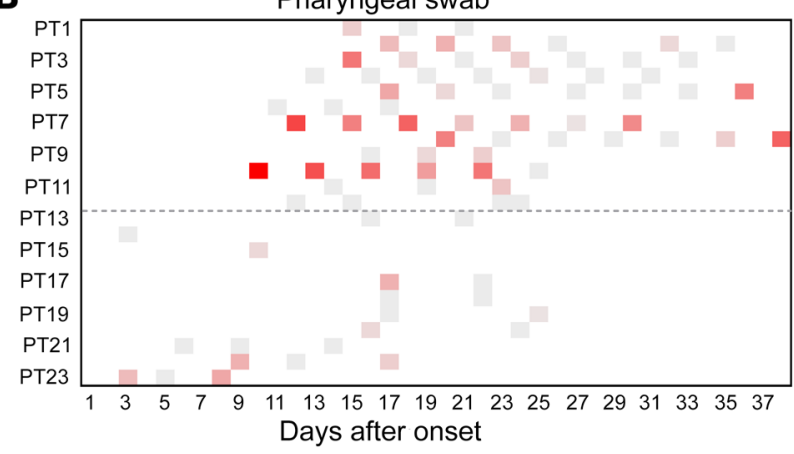

C

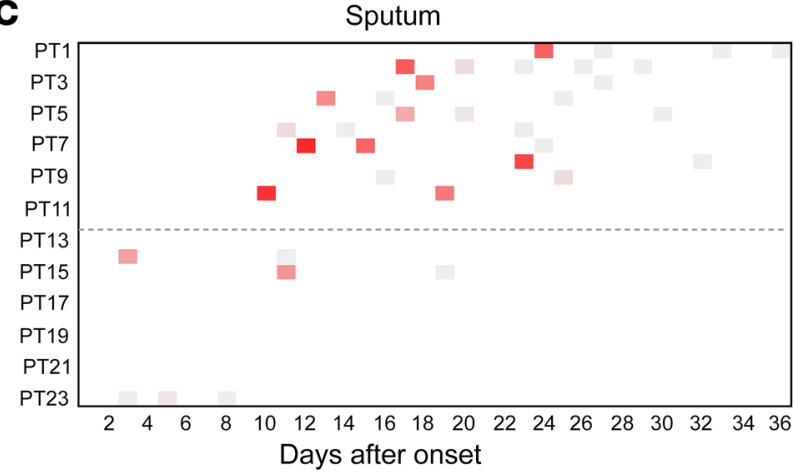

D

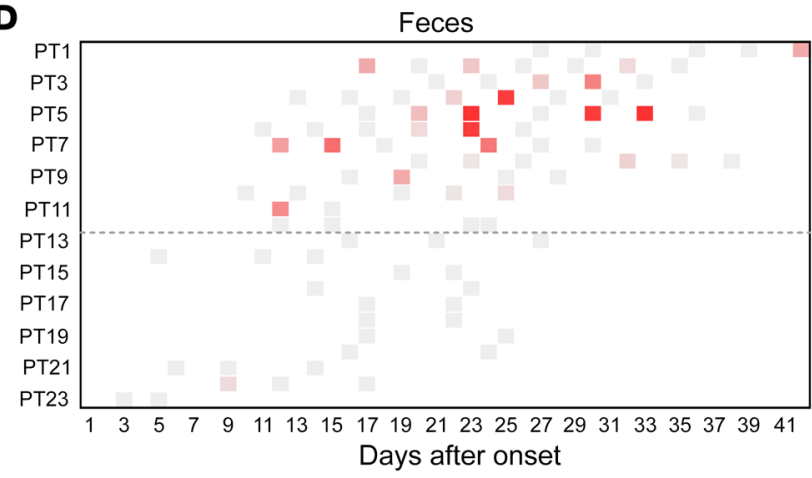

E

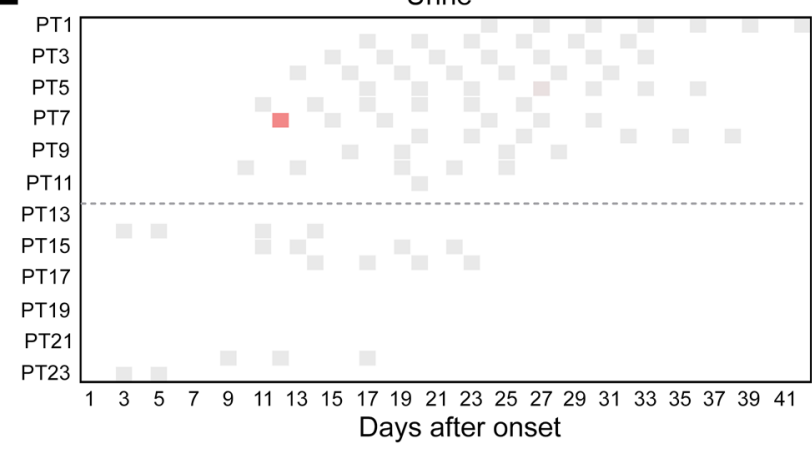

$\mathbf{F}$

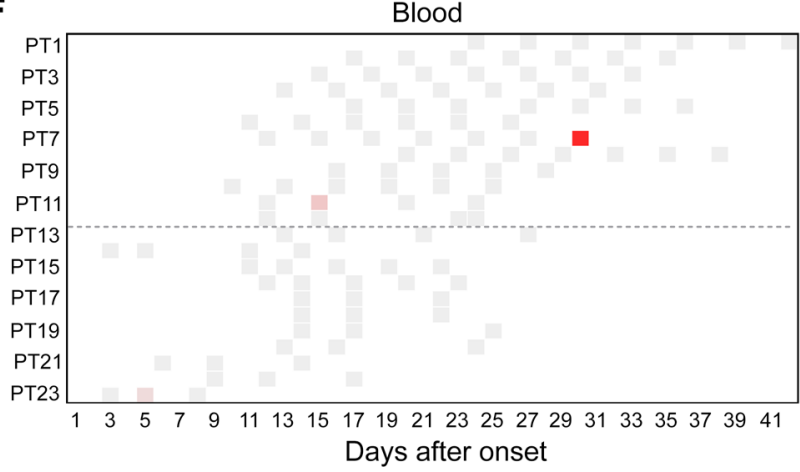

Ct value

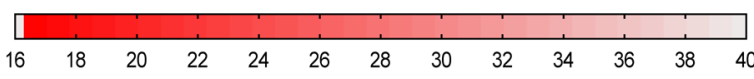

Figure 1. Temporal profile of serial viral load from different tissue samples. Viral loads in patients in the ICU (PT1-PT12) and patients with mild disease (PT13-PT23) as measured by nasal swabs (A), pharyngeal swabs (B), sputum (C), feces (D), urine (E), and blood (F). The $x$ axis indicates the number of days after onset, the $y$ axis indicates patient numbers. Heatmap of $C t$ values of viral loads were shown. A Ct value less than 37 indicates the presence of SARSCoV-2 nucleic acid in the sample. Each square represents 1 sample detected and the gray squares indicate that the sample was viral nucleotide acid-negative.

responses against SARS-CoV-2 in patients, IgM and IgG antibody responses against the $\mathrm{N}$ protein of SARS-CoV-2 in plasma were assayed (Figure 2). One hundred and twenty plasma specimens were obtained from 23 patients at different time points. IgM responses in patients with severe disease increased within 1 to 2 weeks after onset and gradually decreased after 4 weeks (Figure $2 \mathrm{~A}$ ), whereas IgM responses were much lower in mildly ill patients. Most of the mildly ill patients (8/11) did not produce significant IgM antibody throughout the whole disease course, indicating that the IgM diagnosis for mildly ill patients was not sensitive and efficient (Figure 2A). IgG responses emerged at 10-15 days after onset (Figure 2B). Most patients showed high levels of IgG antibodies that were maintained for at least for 6 weeks (Fig- ure 2B). Forty-eight plasma samples collected from healthy volunteer donors (HDs) in 2017-2018 were used as controls to assess the specificity of the tests. As compared with the positive and negative controls, HDs did not have any SARS-CoV-2 specific antibodies.

Detection of IgM and IgG antibodies in respiratory specimens and other body fluids. To investigate the presence of SARS-CoV-2specific antibodies in other tissues with viral shedding, 93 urine, 60 fecal, 67 sputum, 1 BALF, and 1 pleural effusion samples were collected from severely and mildly ill patients. IgM and IgG antibody responses against SARS-CoV-2 nucleocapsid protein were detected. Viral-specific IgM was detected in urine (3/10) and sputum (4/10) in severely ill patients. Viral-specific IgG was present in urine (7/10) and sputum (7/10) in 10 severely ill patients (Figure 


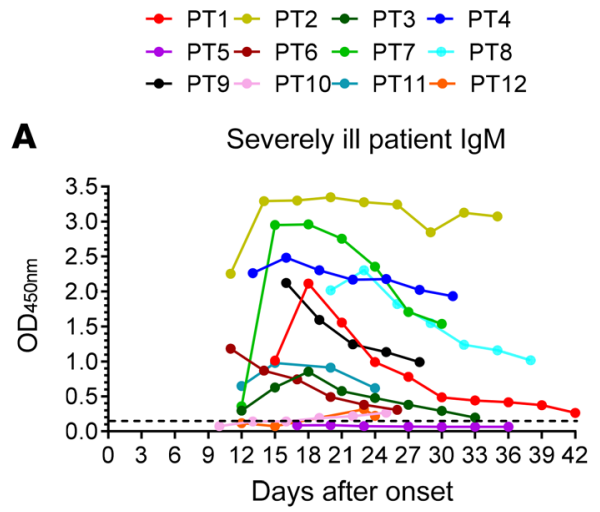

B

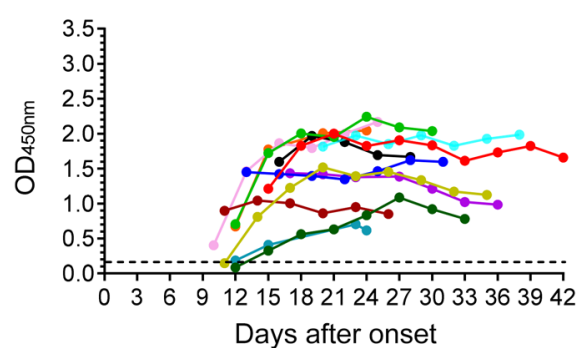

- $\mathrm{PT} 13-\mathrm{PT} 14-\mathrm{PT} 15-\mathrm{PT} 16$

$\leadsto \mathrm{PT} 17-\mathrm{PT} 18=\mathrm{PT} 19 \leadsto \mathrm{PT} 20$

$₫ \mathrm{PT} 21 \pitchfork \mathrm{PT} 22 \_\mathrm{PT} 23$

Mildly ill patient IgM

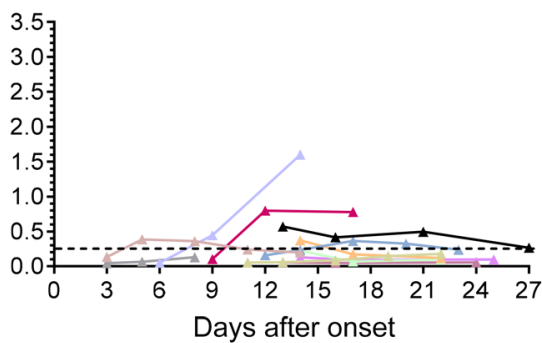

Mildly ill patient $\lg G$

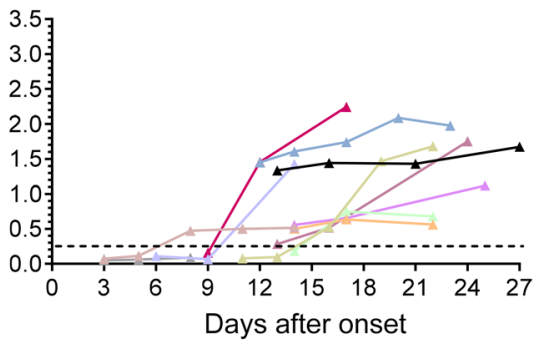

Healthy donor IgM

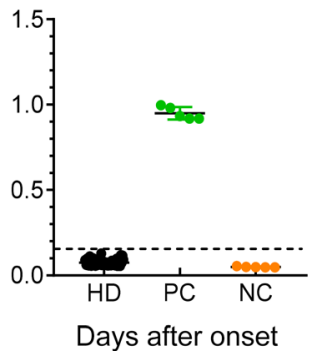

Healthy donor $\lg G$

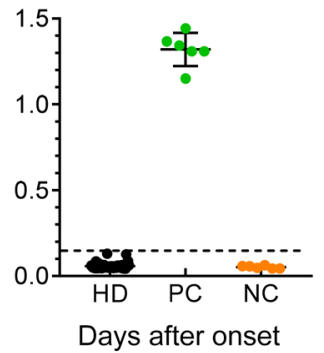

Figure 2. Kinetics of IgM and IgG responses against SARS-CoV-2 in severely and mildly ill patients. IgM (A) and IgG (B) antibody responses against the N protein of SARS-CoV-2 in plasma were detected. Serial plasma samples were collected from 12 severely ill and 11 mildly ill patients infected with SARSCoV-2. Forty-eight plasma samples previously collected from healthy volunteer donors in 2017-2018 were used as a healthy donor group (HD). Positive (PC) and negative (NC) controls provided by detection kit were included to ensure test validity.

3, A and B). In contrast, no antibody was detected in the mildly ill group, indicating that severe infection might result in tissue damage, including to the airways in the lung and to the kidneys (Figure 3, A and B). Detection rates of IgM were lower in the urine and sputum of severely ill patients, which was consistent with the larger size of IgM pentamers than monomer IgG. Appearance of SARS-CoV-2-specific IgG in urine and sputum could be a potential marker to determine disease severity. No antibody was detected in fecal samples (Figure 3C). SARS-CoV-2-specific IgM and IgG antibodies were also detected in bronchoalveolar lavage fluid (BALF) and pleural effusion from the same severe patient (PT7), indicating various tissue damage and antibody distribution in severely ill patients (Figure 3D).

The S2 fragment of spike protein was preferentially recognized by $S A R S-C o V-2-s p e c i f i c$ antibodies in patients. To compare the antigenicity of different SARS-CoV-2 structural proteins, S (aa 1-1213 of spike protein), S1 (aa 1-685 of spike protein), S2 (aa 686-1213 of spike protein), RBD (receptor binding domain, aa 319-514 of spike protein), and $\mathrm{N}$ proteins (aa 1-419 of nucleocapsid protein) were used as coating antigens for ELISAs (Figure 4). All of the S, S1, S2, $\mathrm{RBD}$, and $\mathrm{N}$ proteins were recognized by patient plasma and peaked at 3-4 weeks after onset. The seroconversion rates against $\mathrm{S}$ and S2 proteins reached $100 \%$ between $7-14$ days after illness onset. They were lower against S1 (10/20, 50\%), RBD (13/20, 65\%), and $\mathrm{N}(18 / 20,90 \%)$ proteins at 7-14 days, and reached $100 \% 3$ weeks after illness onset (Figure 4, B, C, and E). ELISAs using S2 and S2-containing full-length $S$ protein performed better than the others and could detect SARS-CoV-2-specific antibody in all patients even at the first 2 weeks after onset (Figure 4, A and D). HDs did not have any SARS-CoV-2-specific antibodies. No obvious differences in IgG response against viral proteins (S, S2, RBD, and N) were observed between severely and mildly ill patients, except in anti-S1 IgG response, due to limited samples. The correlations between IgG levels against different viral proteins (S, S1, S2, N, and $\mathrm{RBD}$ ) were performed and compared as shown in Figure 4F. Most IgG responses against different viral proteins (RBD, N, S, and S1) showed moderate to strong correlations with each other, except for the correlation between anti-S2 IgG and anti-S1/N IgG. In addition, the correlation between anti-S and anti-S2 $\operatorname{IgG}\left(r^{2}=0.6902\right)$ was better than that between anti-S and anti-N IgG $\left(r^{2}=0.4255\right)$.

Strong cross-reactive antibody responses between SARS-CoV-2 and SARS-CoV, but not between SARS-CoV-2 and MERS-CoV. Spike (S) and nucleocapsid (N) proteins of 6 human CoVs were used to establish in-house ELISAs for IgG antibody detection. Ninety-six HDs were included as controls. Plasma from both the severely and mildly ill groups recognized HCoV-229E, NL63, HKU1, and OC43, which shared a similar trend as HDs since these viruses were prevalent worldwide and most adults were serologically positive for these viruses (Figure $5, \mathrm{~A}-\mathrm{D})(15,16)$. To further investigate cross-reactivity with SARS-CoV and MERS-CoV, plasma was obtained in 2018 from 18 SARS-convalescent patients, 15 years after SARS-CoV infection, and in 2015 from 12 MERS-convalescent patients, 6-18 months after MERS-CoV infection, for inclusion in this study. Plasma from SARS-convalescent patients showed high levels of cross-reactivity against SARS-CoV-2 S and $\mathrm{N}$ proteins, as predicted, since SARS-CoV shared $88.6 \%$ with $\mathrm{N}$ 
A
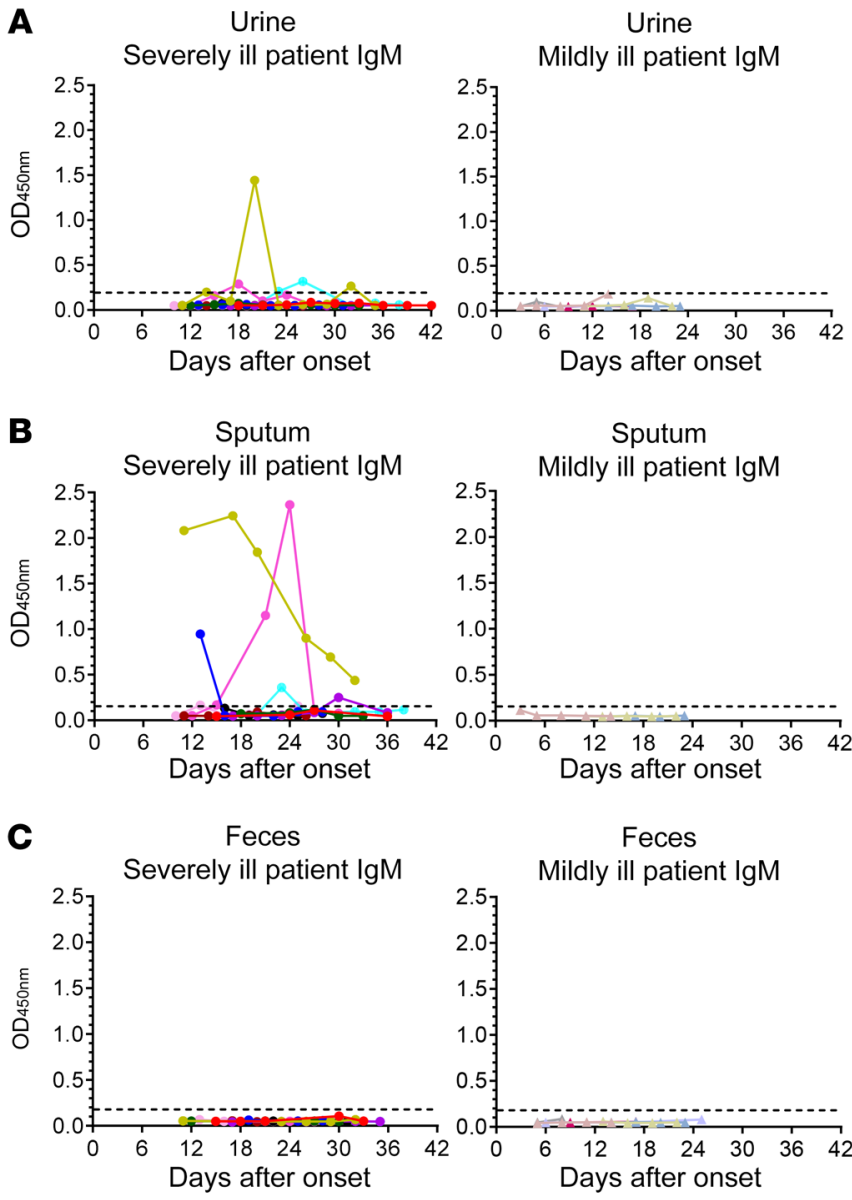

Sputum Mildly ill patient IgM

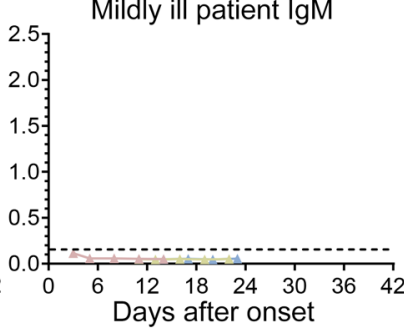

Feces
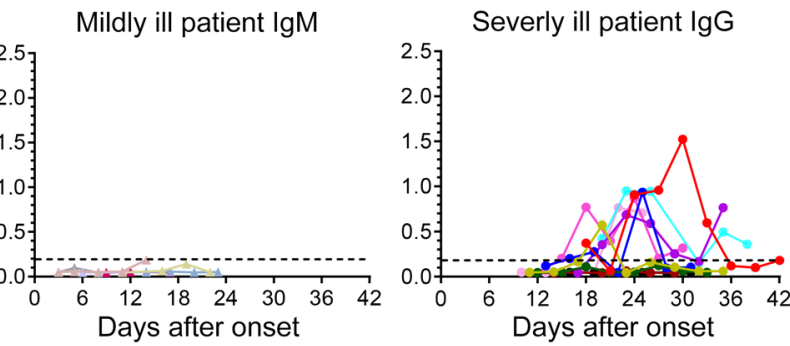

Sputum

Severly ill patient IgG

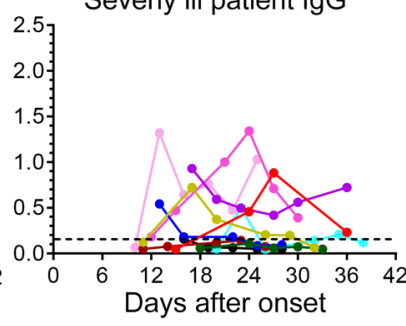

Feces

Severly ill patient IgG
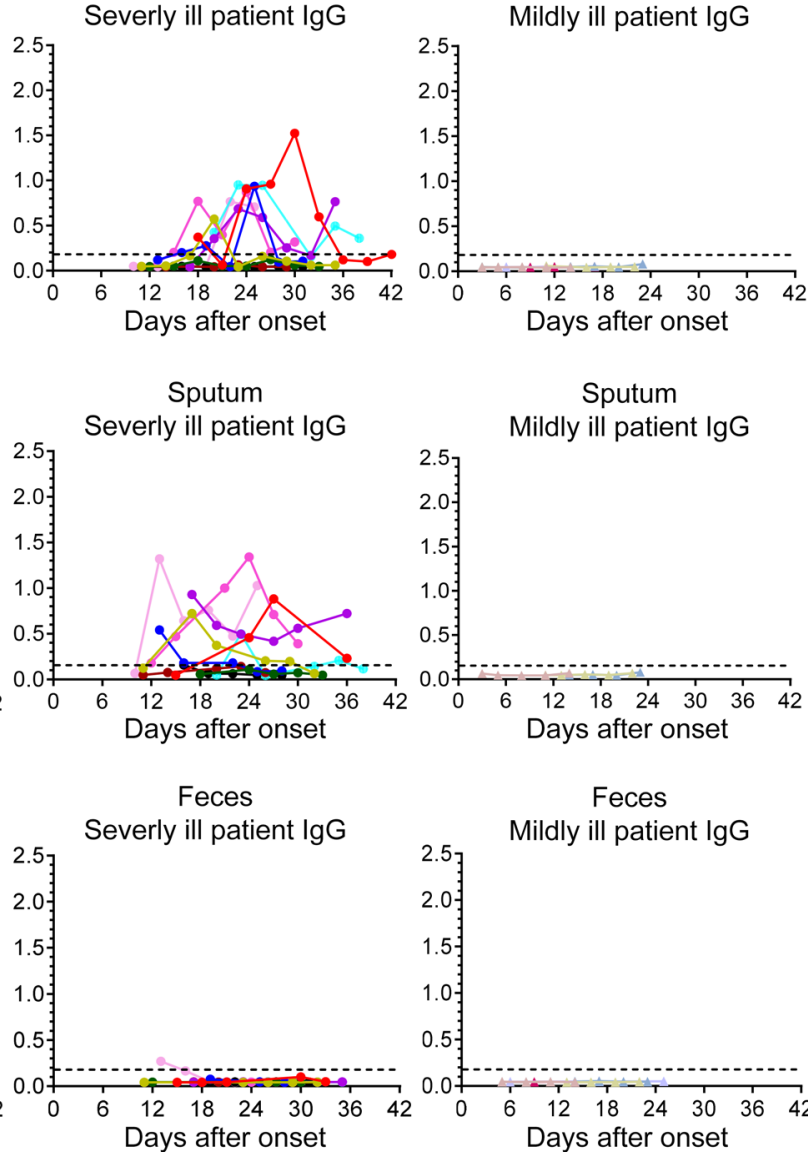

Sputum

Mildly ill patient $\lg G$
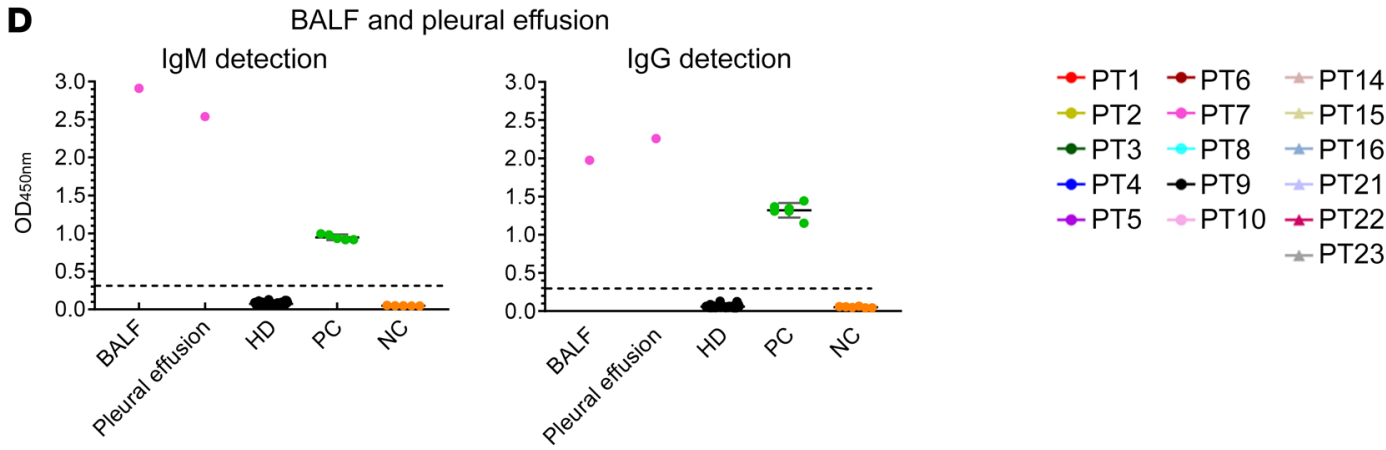

Figure 3. Kinetics of IgM and IgG responses against SARS-CoV-2 in different tissues. Urine (A), sputum (B), feces (C), BALF, and pleural effusion (D) specimens from patients with COVID-19 were detected for the presence of IgM and IgG antibodies against the N protein of SARS-CoV-2. Positive (PC) and negative (NC) controls provided by detection kit were included to ensure test validity. Plasma from 48 HDs was also included.

and 69.2\% with S homologies, respectively (Figure 5E and ref. 17). No obvious cross-reactivity was observed between MERS-CoVand SARS-CoV-2-specific antibodies. MERS-CoV is still circulating in Middle East countries. Patients infected with MERS-CoV showed similar clinical symptoms as those observed in patients with COVID-19. However, our results indicated that doctors were unlikely to misdiagnose patients with MERS and COVID-19 using serological tests (Figure $5 \mathrm{~F}$ ).

Neutralizing activities were correlated with the magnitude of SARSCoV-2 $S$ and $N$ antibody responses. Little is known about the kinetics of anti-SARS-CoV-2-neutralizing antibodies in patients with severe or mild disease. Neutralizing antibodies against authentic SARS-CoV-2 in severely and mildly ill patients were evaluated. We found that $73.9 \%(17 / 23)$ of patients generated robust neutralizing antibodies (50\% focus reduction neutralization test titer [ $\left.\mathrm{FRNT}_{50}\right]$ $>500$ ) 3 weeks after disease onset (Figure 6, A and B). Higher neutralizing antibody titers were induced in the severely ill group as compared with mildly ill patients. Plasma samples collected from patients 3 weeks after onset were also tested to compare their neutralizing activities against the SARS-CoV-2 pseudotype (Figure 6C) and authentic virus (Figure 6D) at a fixed dilution (1:40). Similar results were obtained with both neutralizing tests, indicating there 
A

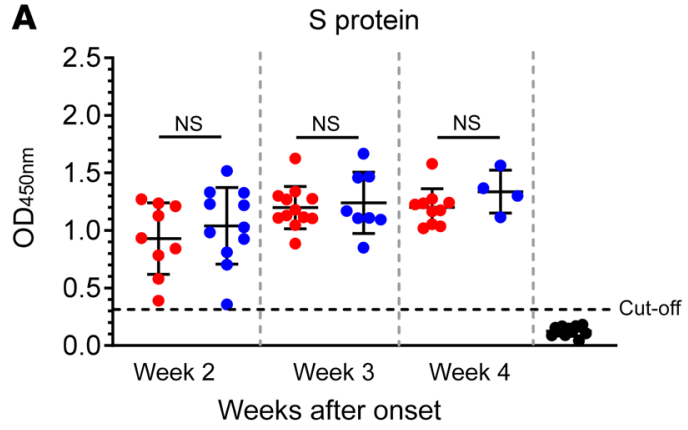

C

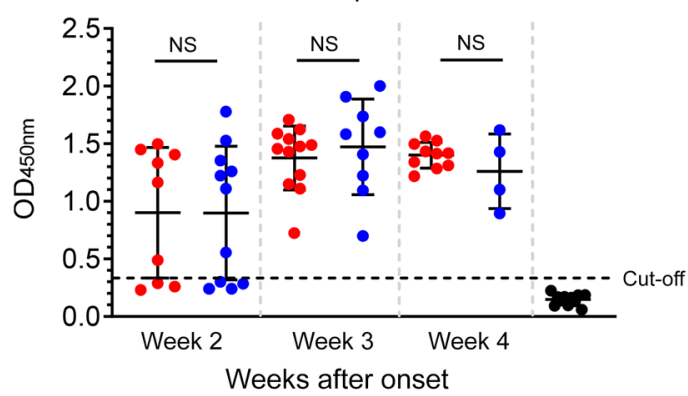

E

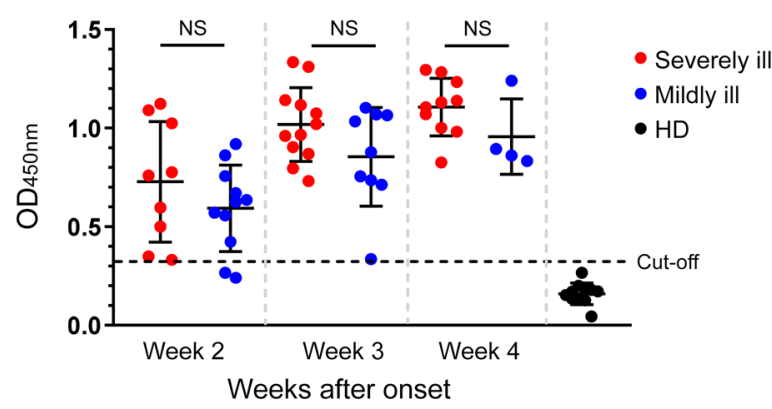

B

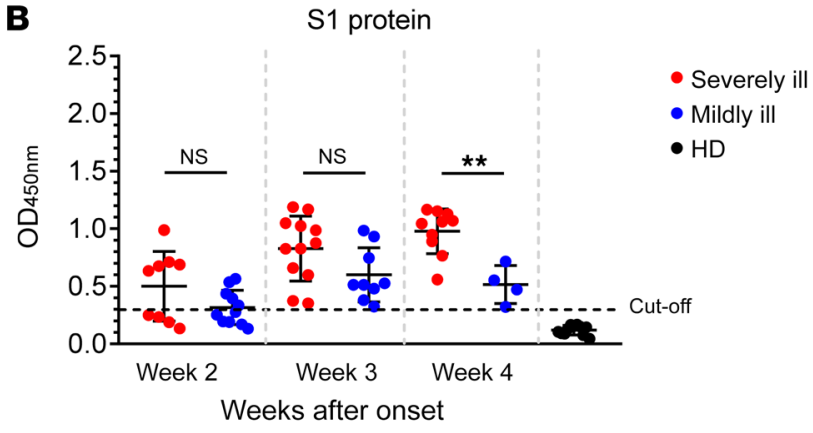

D

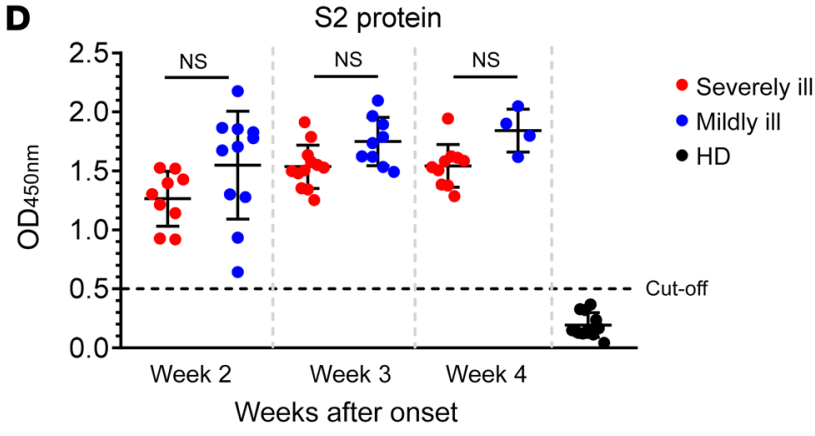

$\mathbf{F}$

\begin{tabular}{|c|c|c|c|c|}
\hline $0.80-1.00$ & \multirow{5}{*}{$\begin{array}{l}\text { Very strong } \\
\text { Strong } \\
\text { Moderate } \\
\text { Weak } \\
\text { Very weak }\end{array}$} & & & \\
\hline $0.60-0.79$ & & & & \\
\hline $0.40-0.59$ & & & & \\
\hline $0.20-0.39$ & & & & \\
\hline $0.00-0.19$ & & & & \\
\hline $\begin{array}{l}r \text { value } \\
(P \text { value })\end{array}$ & s & s1 & RBD & s2 \\
\hline s1 & $\begin{array}{c}0.4554 \\
(0.0005)\end{array}$ & & & \\
\hline RBD & $\begin{array}{c}0.7607 \\
(<0.0001)\end{array}$ & $\begin{array}{c}0.6628 \\
(<0.0001)\end{array}$ & & \\
\hline S2 & $\begin{array}{c}0.6902 \\
(<0.0001)\end{array}$ & $\begin{array}{c}0.0121 \\
(0.9298)\end{array}$ & $\begin{array}{c}0.3751 \\
(0.0048)\end{array}$ & \\
\hline $\mathrm{N}$ & $\begin{array}{c}0.4255 \\
(0.0013)\end{array}$ & $\begin{array}{c}0.8235 \\
(<0.0001)\end{array}$ & $\begin{array}{c}0.6514 \\
(<0.0001)\end{array}$ & $\begin{array}{c}0.1146 \\
(0.4048)\end{array}$ \\
\hline
\end{tabular}

Figure 4. IgG antibody response against different SARS-CoV-2 proteins or fragments. Plasma samples collected at different time points after admission were used for IgC detection in different protein-coated ELISAs: S (1209 aa) (A), S1 (681 aa) (B), RBD (457 aa) (C), S2 (539 aa) (D), and N (430 aa) (E). Eleven plasma samples from HDs were used as controls. The correlations among IgG levels against different viral proteins were analyzed and summarized. Pearson's correlation coefficient was used to assess the relationship among antiviral lgG levels of different proteins (F). A Student's $t$ test was used to analyze differences in mean values between groups A-E. A $P$ value less than 0.05 was considered to be statistically significant. ${ }^{* *} P \leq 0.01$.

was a good correlation between these 2 detection methods $(21 / 23$ pseudotype, 19/23 live virus; focus reduction neutralization test [FRNT]). Plasma from SARS- and MERS-convalescent patients could not neutralize SARS-CoV-2 pseudotype and authentic virus, indicating that SARS- and MERS-convalescent patients could still be vulnerable to SARS-CoV-2 infection (Figure 6, C and D). AntiSARS-CoV-2 $\mathrm{S}$ and $\mathrm{N}$ IgG levels exhibited a moderate correlation with neutralization titers of patients' plasma (Pearson $r=0.5393, P$ $<0.0001$ for $\mathrm{S} ; r=0.6709, P<0.0001$ for $\mathrm{N}$ ), suggesting that monitoring $\mathrm{S}$ and $\mathrm{N}$ antibody levels could be useful to determine neutralizing titer before convalescent plasma transfusion (Figure 6, E and F). No obvious correlation between viral load and neutralizing titer was observed (Figure 6G).

\section{Discussion}

Temporal profile of serial viral loads from different tissue samples of patients indicated that viral shedding was more common in respiratory and fecal material, especially in severely ill patients, and to a much less extent in urine and blood. A recent study reported that detectable SARS-CoV-2 viral RNA in blood strongly correlated with clinical severity (18).

A lower level of IgM response was observed in mildly ill patients than in severely ill patients. The lower level of IgM response associated with mild disease probably reflects lower viral loads and viral antigens. Similar IgG responses were detected in both severely and mildly ill patients. Remarkably, virus-specific $\operatorname{IgM}$ and $\operatorname{IgG}$ were detectable in serial urine and sputum samples 
A

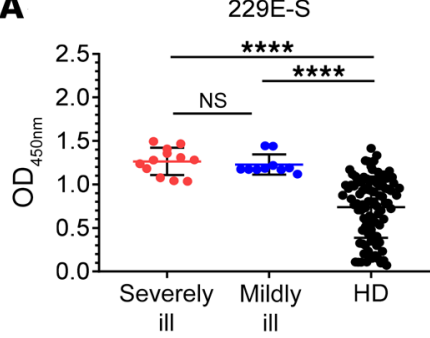

C

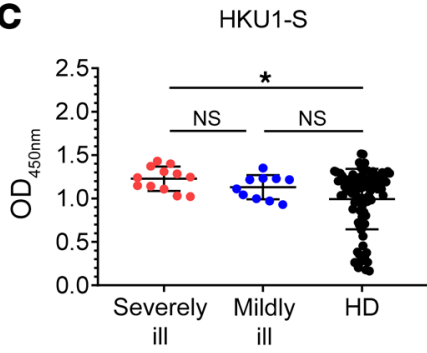

E

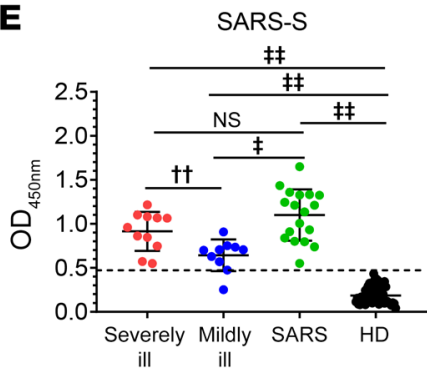

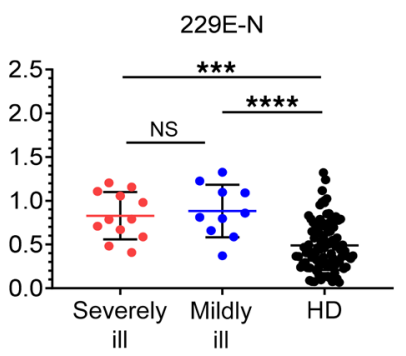

B
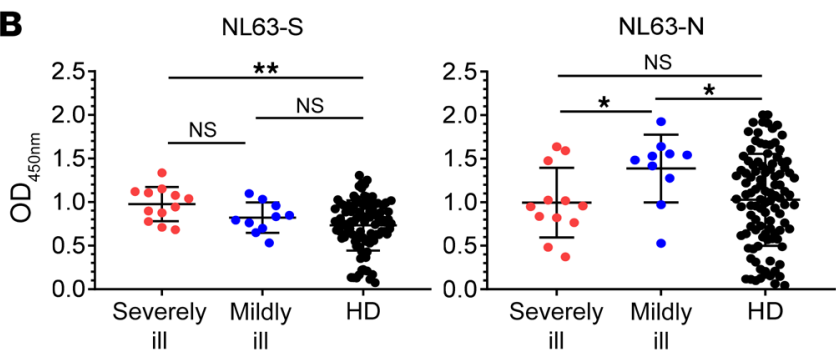

D
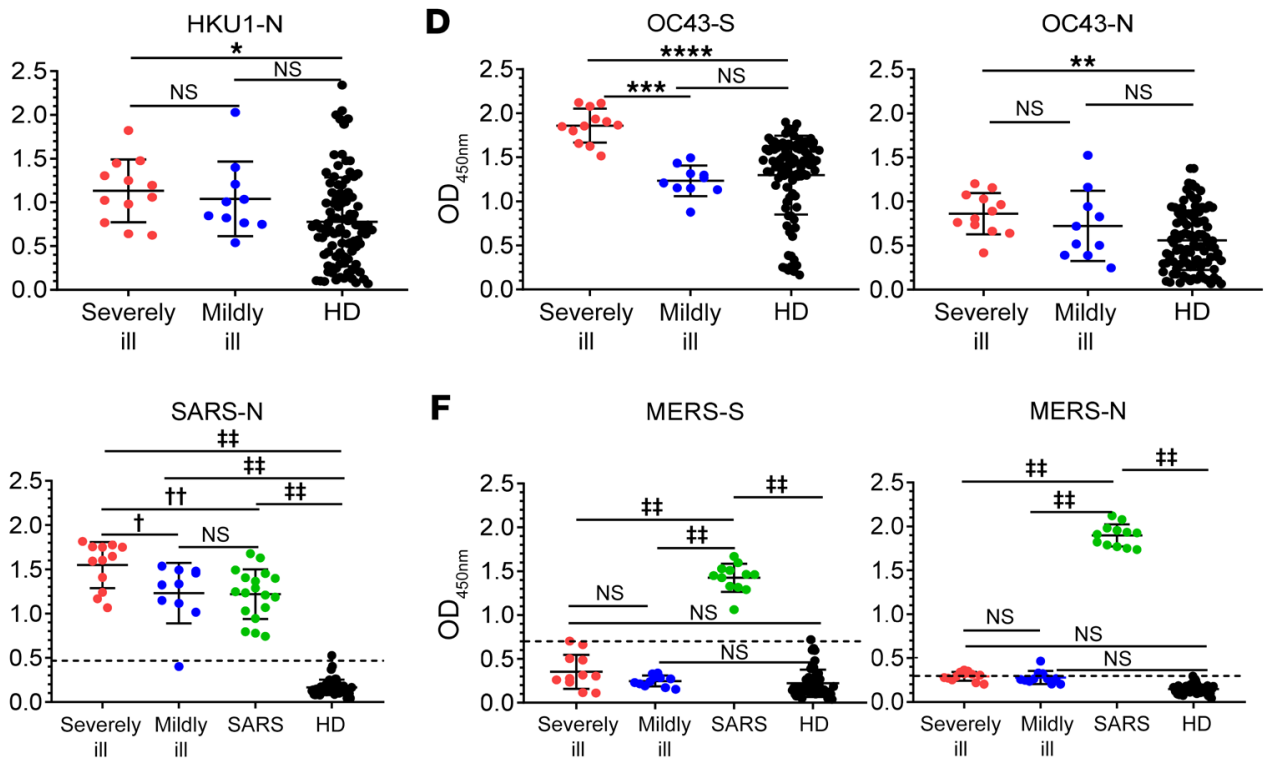

$\mathbf{F}$
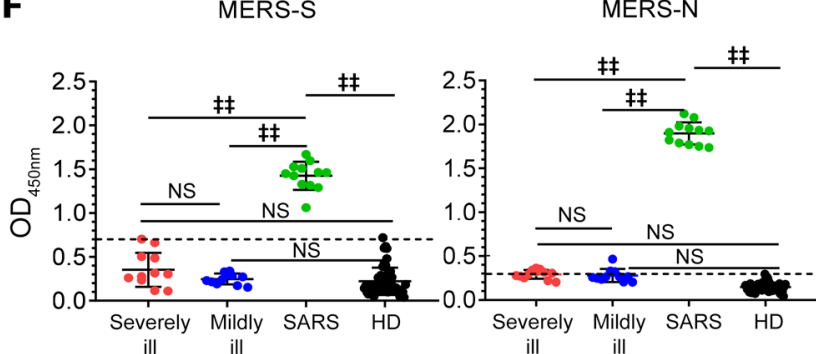

Figure 5. IgC cross-reactivity analysis between the other 6 human CoVs and SARS-CoV-2. Spike (S) and nucleoprotein (N) of the other 6 human CoVs were used as coated target antigens to establish an in-house ELISA to detect IgG antibody for HCoV-229E (A), HCoV-NL63 (B), HCoV-HKU1 (C), HCoV-OC43 (D), SARS-CoV (E), and MERS-CoV (F). Plasma from 96 HDs and 23 SARS-CoV-2-infected patients were used (A-F). Severe indicates a severely ill patient with COVID-19; mild indicates a mildly ill patient with COVID-19; HD indicates healthy donors. Plasma samples from 18 SARS-convalescent (E) and 12 MERS-convalescent (F) patients were used as controls, respectively. A Student's $t$ test was used to analyze differences in mean values between groups (A-F). Experiments for each virus were independently carried out. Multiple comparisons following 1-way ANOVA and Kruskal-Wallis test were performed for statistical analysis. Bonferroni's correction was used to avoid inflation of experiment-wise Type I error. In A-D, a difference was considered statistically significant when the $P$ value was lower than $0.0167(0.05 / 3) ;{ }^{*} P \leq 0.0167,{ }^{* *} P \leq 0.0033,{ }^{* * *} P \leq 0.00033$, ${ }^{* * * *} P \leq 0.000033$. In $\mathbf{E}$ and $\mathbf{F}$, a difference was considered statistically significant when the $P$ value was lower than $0.0083(0.05 / 6) ;{ }^{\dagger} P \leq 0.0083,{ }^{\dagger \dagger} P \leq 0.0017,{ }^{\ddagger} P \leq 0.00017,{ }^{\ddagger \ddagger} P \leq 0.000017$.

of most severely ill patients, but not in mildly ill patients, indicating severe tissue damage in these patients, which could be used as a marker to determine disease severity.

Several SARS-CoV-2 proteins induced IgG responses in severely and mildly ill patients. Although all proteins, including S, S1, S2, RBD, and N, could be used to detect antibody response, $\mathrm{S} 2$ and S2-containing full-length $\mathrm{S}$ proteins performed better in the ELISAs, and antibodies could be detected in most infected patients in the first 2 weeks after onset. The sensitivity of the detection method was associated with abundance, conservation, and antigenicity of viral proteins, indicating that the $\mathrm{S} 2$ region possessed more epitopes recognized by viral-specific antibodies.

Six human coronaviruses have been identified. Although SARS-CoV disappeared, HCoV-229E, NL63, HKU1, and OC43 are circulating worldwide and MERS-CoV primarily is in Middle East countries $(19,20)$. Antigenic cross-reactivity was observed previously between SARS-CoV and HCoV-229E, OC43 (21). Cross-reactivity analysis between SARS-CoV-2 and the other 6 CoVs provided essential information for diagnosis and epide- miological studies, and helped to dissect the roles of preexisting antibodies against other CoVs in patients infected with SARSCoV-2 or MERS-CoV. Our ELISA results showed that although our healthy controls were often infected with 229E, NL63, or HKU1 of OC43 viruses, there was no cross-reaction in SARS-CoV-2, indicating that the SARS-CoV-2 S, S1, RBD, S2, and N protein assays are specific for the virus. Our results showed that most of the patients infected with SARS-CoV-2 were previously infected with the 4 low-pathogenic CoVs. Plasma from patients with COVID-19 also showed a high level of antibody binding to SARS-CoV N and S proteins, which was consistent with high homology between the genomes of these 2 viruses. Of note, stronger cross-reactivities against SARS-CoV proteins were observed in severely ill patients. Although SARS-CoV-2 and HCoV-OC43 full-length spike proteins share relatively low homology $(27.2 \%)$, some regions in S2 fragments showed high homology (70\%-80\%), which might account for the cross-reactivity between HCoV-OC43 and SARS-CoV-2. Lack of obvious cross-reactivity between MERS-CoV and SARSCoV-2 plasma ruled out the possibility of misdiagnosis using sero- 
A

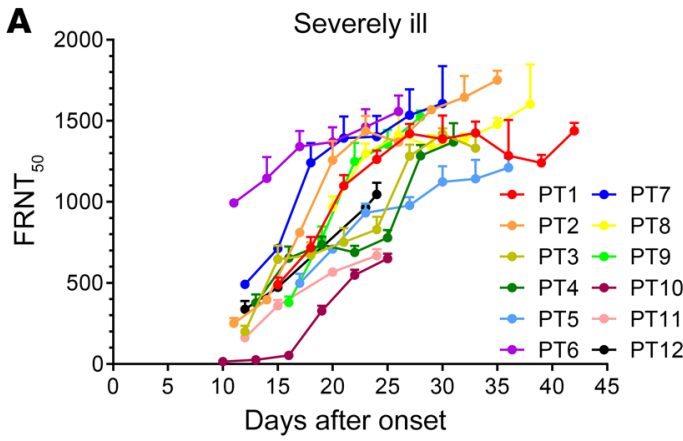

C

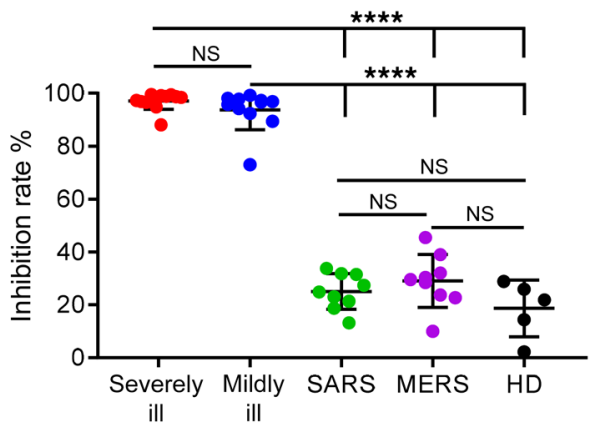

B

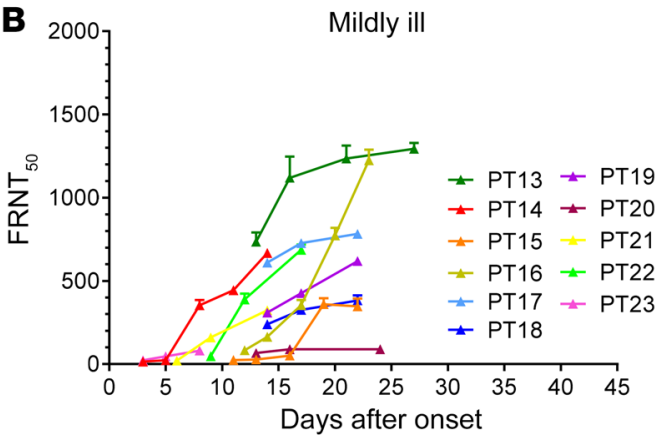

D

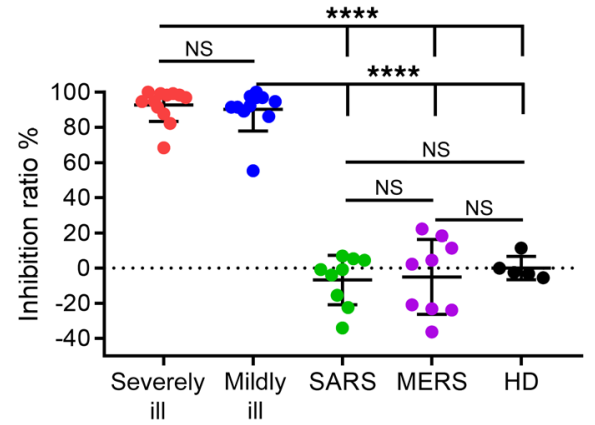

E

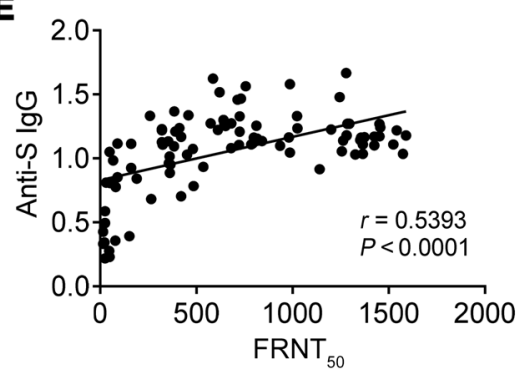

$\mathbf{F}$

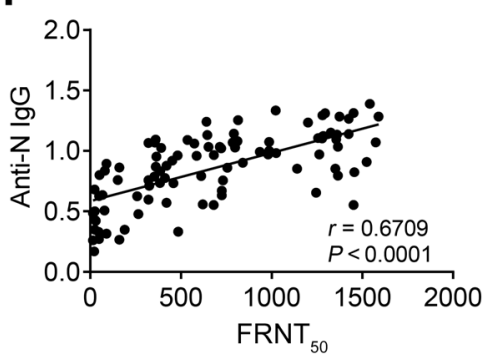

G

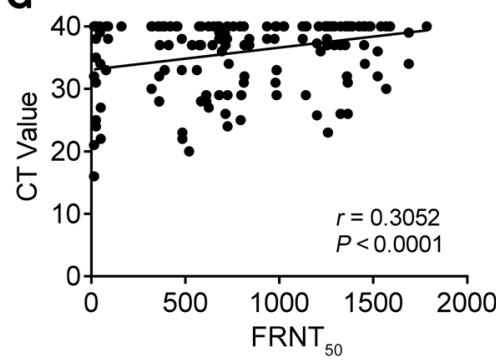

Figure 6. Neutralizing and cross-protection of antibody response against SARS-CoV-2 in severely and mildly ill patients. Serial plasma samples were

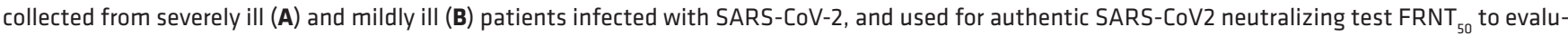
ate kinetics of neutralizing antibodies in SARS-CoV-2 infected patients. Plasma samples collected 3 weeks after onset were used to compare crossneutralizing antibodies between severely ill and mildly ill patients with SARS-CoV-2 and SARS-CoV-convalescent patients using SARS-CoV-2 pseudotype (C) and authentic virus (D) at a fixed dilution (1:40). A Student's $t$ test was used to analyze differences in mean values between groups. Experiments for each virus were independently carried out. Multiple comparisons following 1-way ANOVA and Kruskal-Wallis tests were performed for statistical analysis. Bonferroni's correction was used to avoid inflation of experiment-wise Type I error. There were a total of 10 pairwise comparisons among 5 groups. Hence, a difference was considered statistically significant when the $P$ value was lower than $0.005(0.05 / 10)$. ${ }^{* * * *} P \leq 0.0001(\mathbf{C}$ and $\mathbf{D})$. Pearson's correlation coefficient was used to assess the relationship between neutralizing titer and S- and N-specific IgC levels (E and F); viral loads of respiratory specimens (G) were analyzed.

logic tests in the Middle East region, where MERS-CoV has been prevalent for more than 7 years.

The plaque/focus reduction neutralization assay is considered to be the gold standard of quantity antibody neutralization titer. In this study, the SARS-CoV-2 pseudotype neutralization system and the FRNT assay were compared. SARS-CoV-2 pseudotype expressing the SARS-CoV-2 spike protein was successfully used to detect neutralizing antibody with similar sensitivity, which could be useful for hospitals without Biosafety Level 3 (BSL-3) labs where they could do a neutralizing test before convalescent plasma transfusion. In addition, anti-SARS-CoV-2 S and N IgG levels exhibited moderate correlation with neutralization titers of patients' plasma, which also provided an alternative method to determine neutralizing titers. Plasma from convalescent SARS and MERS patients could not inhibit the SARS-CoV-2 pseudotype and authentic virus entry. Although SARS-CoV shared 88.6\% homology with SARS-CoV-2, a recent study showed that their RBD, which is the major domain for neutralizing antibody induction, was different in structure, as observed under CryoEM (22).

In summary, this study provides comprehensive information on kinetics, tissue distribution, cross-reactivities, and neutralization of antibody responses in patients with COVID-19. It will improve our understanding of humoral immune response in humans after SARS-CoV-2 infection and shed light on diagnosis, prognosis, convalescent plasma transfusion therapy, and epidemiology studies of SARS-CoV-2 infection in humans. 


\section{Methods}

Patient enrollment and sample collection. Between January 28, 2020, and February 24, 2020, 23 patients with novel coronavirus (SARS-CoV-2) infection confirmed by real-time PCR were hospitalized in the First Affiliated Hospital of Guangzhou Medical University ( $n=13$ patients), the Sixth Affiliated hospital of Guangzhou Medical University, Qingyuan People's Hospital ( $n=3$ patients), and Yangjiang People's Hospital ( $n=7$ patients). Respiratory swabs, sputum, body fluids, and plasma specimens were collected every 3 to 4 days following admission. Clinical data, including patient demographic information and clinical outcome, were retrieved from the medical records. A total of 12 severely ill and 11 mildly ill patients were enrolled for serological analysis. Patients with severe pneumonia who were admitted to the ICU and required mechanical ventilation were enrolled in the severely ill group; patients with a mild clinical presentation (mainly with fever, cough, malaise, and headache, including nonpneumonia or mild pneumonia) were enrolled in the mildly ill group. Plasma samples from 18 SARS-convalescent patients were collected in 2018 from health care workers who were infected with SARS-CoV 17 years before the present study. Plasma samples from 12 MERS-convalescent patients were acquired as previously described (23). Ninety-six healthy donor plasma samples, collected in 2017-2018, were used as controls in this study.

Real-time PCR detection of SARS-CoV-2. Nucleic acid was extracted from respiratory samples and urine using a Viral RNA extraction kit from Zybio Inc. RNA extraction from feces and blood was performed using a total RNA extraction kit from Sangon Biotech. A real-time PCR assay kit targeting the SARS-CoV-2 RdRp and N gene regions was provided by Zybio Inc. Serial sampling of nasal swabs, throat swabs, sputum, anal swabs, urine, and blood was used to monitor viral shedding.

IgM and IgG ELISAs. Clinical samples of plasma, urine, sputum, feces, BALF, and pleural fluid were collected and analyzed for the presence of SARS-CoV-2-specific IgM and IgG antibodies to the SARS-CoV-2 nucleoprotein using a commercial antibody detection kit provided by Lizhu Medicine Group Holding Co. Ltd. Plasma and urine were used directly in ELISAs whereas feces and sputum were diluted with the same volume of PBS and centrifuged at $900 \mathrm{~g}$ for 10 minutes before supernatant was harvested for antibody detection. According to the manufacturer's instructions, the threshold IgM value was negative control plus 0.1, whereas the threshold IgG value for the presence of SARS-CoV-2 infection was negative control plus 0.13.

Comparison of antibody response to different SARS-CoV-2 proteins. To assess the antibody response to different SARS-CoV-2 proteins or different fragments of the spike protein, SARS-CoV-2 S (spike protein, 1203 aa), S1 (675 aa), S2 (533 aa), RBD (228 aa), and N (424 aa) proteins were obtained from Sino Biological Inc. and in-house ELISAs for detection of SARS-CoV-2-specific IgG antibody were established. Briefly, 96-well plates (Jet Biofil Co. Ltd.) were coated with $100 \mu \mathrm{L} /$ well $(0.5 \mu \mathrm{g} / \mathrm{mL})$ SARS-CoV-2 S, S1, S2, RBD, or N protein in DPBS buffer (Thermo Fisher Scientific) overnight at $4^{\circ} \mathrm{C}$. After blocking (DPBS, $10 \% \mathrm{FBS}$ ), $100 \mu \mathrm{L}$ diluted plasma (1:100) was added and plates were incubated at $37^{\circ} \mathrm{C}$ for 1 hour. After washing, plates were incubated with $100 \mu \mathrm{L}$ HRP-conjugated mouse anti-human IgG $(\mathrm{H}+\mathrm{L})$ antibody (Jackson ImmunoResearch, catalog 109-035-088) at $37^{\circ} \mathrm{C}$ for 1 hour. Reactions were visualized by adding $50 \mu \mathrm{L}$ TMB substrate solution (Biohao Biotechnology Co. Ltd.). Optical densities at $450 \mathrm{~nm}$ were then read. The mean value of healthy donor anonymous plasma (HD group) collected in 2017-2018 plus 3 standard deviations was used as the detection threshold.
Cross-reactivity between SARS-CoV-2 and the other 6 human CoVs. To determine the cross-reactivity between SARS-CoV-2 and the other 6 human respiratory CoVs (SARS-CoV, MERS-CoV, HCoV-NL63, HCoV-OC43, HCoV-229E, and HCoV-HKU1), we purchased S and N proteins of all 6 human CoVs from Sino Biological Inc. and established an in-house viral protein ELISA for cross-reactive $\operatorname{IgG}$ antibody detection as described above.

Pseudotype-based neutralization assay. To safely and rapidly assess the neutralization activity of patients' plasma against SARS-CoV-2, we developed a luciferase reporter-based pseudotype neutralization assay that has a nonreplicative human immunodeficiency virus backbone coated with the SARS-CoV-2 spike protein. SARS-CoV-2 pseudotype neutralization assays were performed on ACE2-overexpressing HEK293 cells (ATCC) (HEK293-ACE2 cells) in 96-well microplates. Fifty microliters of 20-fold-diluted patients' plasma was combined with an equal volume of the SARS-CoV-2 pseudotype and incubated for 60 minutes at $37^{\circ} \mathrm{C}$. The mixtures were then added to 96-well plates seeded with HEK293-ACE2 cells at $3.8 \times 10^{4}$ cell/well. Cells were further cultured for 40 hours at $37^{\circ} \mathrm{C}$. Luciferase activity in cell lysates was measured using the steady-Glo luciferase assay kit (Promega). Neutralizing activity was defined as the ratio of inhibition of SARS-CoV-2 pseudotype (SARS-CoV-2-pp) luciferase activity comparing patients' plasma (1:40 dilution) to control.

Focus reduction neutralization test. SARS-CoV-2 FRNT was performed in a certified BSL-3 lab. Fifty microliters of plasma samples were serially diluted, mixed with $50 \mu \mathrm{L}$ SARS-CoV-2 (100 focus forming units [FFU]) in 96-well microwell plates, and incubated for 1 hour at $37^{\circ} \mathrm{C}$. Mixtures were then transferred to 96 -well plates seeded with Vero E6 cells (ATCC) for 1 hour at $37^{\circ} \mathrm{C}$ to allow virus entry. Inoculums were removed before adding the overlay media $(100 \mu \mathrm{L}$ MEM containing $1.2 \%$ carboxymethylcellulose). The plates were then incubated at $37^{\circ} \mathrm{C}$ for 24 hours. Overlays were removed and cells were fixed with $4 \%$ paraformaldehyde solution for 30 minutes. Cells were permeabilized with $0.2 \%$ Triton $\mathrm{X}-100$ and incubated with cross-reactive rabbit antiSARS-CoV-N IgG (Sino Biological Inc., catalog 40143-R001) for 1 hour at room temperature before adding HRP-conjugated goat anti-rabbit IgG (H+L) antibody (1:4000 dilution) (Jackson ImmunoResearch, catalog 111-035-144). Cells were further incubated at room temperature. The reactions were developed with KPL TrueBlue Peroxidase substrates (Seracare Life Sciences Inc.). The number of SARS-CoV-2 foci was calculated using an EliSpot reader (Cellular Technology Ltd.).

Statistics. Statistical analysis was performed using GraphPad Prism software, version 7.00. Pearson's correlation coefficient was used to assess the relationship between antiviral IgG levels and neutralizing titers. A Student's $t$ test was used to analyze differences in mean values between groups. Cut-off values are assigned for evaluation of significance of the $P$ value according to different statistical analysis methods indicated in each figure legends. All values are depicted as mean \pm SEM. Multiple comparisons following 1-way ANOVA and Kruskal-Wallis tests were performed for statistical analysis in cross-reactivity and neutralizing experiments; experiments for each virus were independently carried out. Bonferroni's correction was used to avoid inflation of experiment-wise Type I error.

Study approval. This study had IRB approval from the Health Commission of Guangdong Province as well as the ethics committees of each of the hospitals used to obtain patient and healthy donor samples. Written informed consent was obtained from all participants. 


\section{Author contributions}

Jincun Zhao, Yimin Li, JSMP, and Jingxian Zhao conceived the study; YW, LZ, LS, FY, SR, BZ, TS, ANA, RC, Z Zhang, MG, AZ, YH, and LL collected clinical specimens and executed the experiments; CKPM, MMAG, HT, ZL, XH, FL, JS, YZ, LW, Yuming Li, ZC, Z Zhuang, J Zhuo, CC, LK, JW, HL, YJ, ML, Y Lin, YD, LT, and JL analyzed the data; NZ, SP, and JH contributed to critical revision of the manuscript; Jincun Zhao, JSMP, YW, and LZ wrote the manuscript. All authors revised and approved the final version.

\section{Acknowledgments}

The use of patients' specimens for this study was approved by the Health Commission of Guangdong Province. This study was funded by grants from The National Key Research and Development Program of China (2018YFC1200100, 2018ZX10301403), emergency grants for prevention and control of SARS-CoV-2 from the Ministry of Science and Technology (2020YFC0841400) and Guangdong province (2020B111108001, 2018B020207013, 2020B111112003), the Guangdong Province Basic and Applied Basic Research Fund (2020A1515010911), the National Key Technology R\&D Program (2018YFC1311900), the Guangdong Science and Technology Foundation (2019B030316028), the State Key Laboratory of Respiratory Disease (SKLRD-QN-201715, SKLRD-QN-201912 and SKLRD-Z-202007), the Guangzhou Medical University High-Level University Innovation Team Training Program (Guangzhou Medical University released 2017, no. 159), the NIH (P01 060699 to SP), the National Natural Science Foundation of China (NSFC)/Research Grants Council (RGC) Joint Research Scheme (N_HKU737/18), and the Research Grants Council of the Hong Kong Special Administrative Region, China (project no. T11-712/19-N). We thank the patients who took part in this study. We thank Eric Lau at the HKU-Pasteur Research Pole, School of Public Health, University of Hong Kong, for helping with statistical analysis.

Address correspondence to: Jincun Zhao, State Key Laboratory of Respiratory Disease, National Clinical Research Center for Respiratory Disease, Guangzhou Institute of Respiratory Health, First Affiliated Hospital of Guangzhou Medical University; Institute of Infectious Disease, Guangzhou Eighth People's Hospital of Guangzhou Medical University, 195 Dongfeng Xi Road, Yuexiu District, Guangzhou, China 510120. Phone: 86.20.83205059; Email: zhaojincun@gird.cn. Or to: Yimin Li, State Key Laboratory of Respiratory Disease, National Clinical Research Center for Respiratory Disease, Guangzhou Institute of Respiratory Health, the First Affiliated Hospital of Guangzhou Medical University, 195 Dongfeng Xi Road, Yuexiu District, Guangzhou, China 510120. Phone: 86.20.83062114; Email: dryiminli@vip.163.com. Or to: Peiris Malik, HKU-Pasteur Research Pole, School of Public Health, Li Ka Shing Faculty of Medicine, University of Hong Kong; L6-42, Laboratory Block, 21 Sassoon Road, Hong Kong SAR, China 19406. Phone: 852.3917.7537; Email: malik@hku.hk. Or to: Jingxian Zhao, State Key Laboratory of Respiratory Disease, National Clinical Research Center for Respiratory Disease, Guangzhou Institute of Respiratory Health, First Affiliated Hospital of Guangzhou Medical University, 195 Dongfeng Xi Road, Yuexiu District, Guangzhou, China 510120. Phone: 86.20.83205061; Email: zhaojingxian@gird.cn.
1. Almeida JD, Tyrrell DA. The morphology of three previously uncharacterized human respiratory viruses that grow in organ culture. J Gen Virol. 1967;1(2):175-178.

2. Pyrc K, Berkhout B, van der Hoek L. The novel human coronaviruses NL63 and HKU1. J Virol. 2007;81(7):3051-3057.

3. Schmidt OW, Kenny GE. Immunogenicity and antigenicity of human coronaviruses $229 \mathrm{E}$ and OC43. Infect Immun. 1981;32(3):1000-1006.

4. van der Hoek L, et al. Croup is associated with the novel coronavirus NL63. PLoS Med. 2005;2(8):e240.

5. Peiris JS, Yuen KY, Osterhaus AD, Stöhr K. The severe acute respiratory syndrome. $\mathrm{N} \mathrm{Engl} \mathrm{JMed}$. 2003;349(25):2431-2441.

6. Zaki AM, van Boheemen S, Bestebroer TM, Osterhaus AD, Fouchier RA. Isolation of a novel coronavirus from a man with pneumonia in Saudi Arabia. N Engl J Med. 2012;367(19):1814-1820.

7. Zhu N, et al. A novel coronavirus from patients with pneumonia in China, 2019. N Engl J Med. 2020;382(8):727-733.

8. Lai CC, Shih TP, Ko WC, Tang HJ, Hsueh PR. Severe acute respiratory syndrome coronavirus 2 (SARS-CoV-2) and coronavirus disease-2019 (COVID-19): The epidemic and the challenges. Int J Antimicrob Agents. 2020;55(3):105924.

9. Chan JF, et al. A familial cluster of pneumonia associated with the 2019 novel coronavirus indicating person-to-person transmission: a study of a family cluster. Lancet. 2020;395(10223):514-523.

10. European Centre for Disease Prevention and Control. COVID-19 situation update worldwide, as of 20 August 2020. https://www.ecdc.europa.eu/en/ geographical-distribution-2019-ncov-cases.

11. Zhou P, et al. A pneumonia outbreak associated with a new coronavirus of probable bat origin. Nature. 2020;579(7798):270-273.

12. Chen $\mathrm{N}$, et al. Epidemiological and clinical characteristics of 99 cases of 2019 novel coronavirus pneumonia in Wuhan, China: a descriptive study. Lancet. 2020;395(10223):507-513.

13. Wang D, et al. Clinical characteristics of 138 hospitalized patients with 2019 novel coronavirusinfected pneumonia in Wuhan, China [published online February 7, 2020]. JAMA. https://doi. org/10.1001/jama.2020.1585.

14. Guan WJ, et al. Clinical characteristics of coronavirus disease 2019 in China. N Engl J Med. 2020;382(18):1708-1720.

15. Severance EG, et al. Development of a nucleocapsid-based human coronavirus immunoassay and estimates of individuals exposed to coronavirus in a U.S. metropolitan population. Clin Vaccine Immunol. 2008;15(12):1805-1810.

16. Su S, et al. Epidemiology, genetic recombination, and pathogenesis of coronaviruses. Trends Microbiol. 2016;24(6):490-502.

17. Lu R, et al. Genomic characterisation and epidemiology of 2019 novel coronavirus: implications for virus origins and receptor binding. Lancet. 2020;395(10224):565-574.

18. Chen W, et al. Detectable 2019-nCoV viral RNA in blood is a strong indicator for the further clinical severity. Emerg Microbes Infect. 2020;9(1):469-473.

19. Lee J, Storch GA. Characterization of human coronavirus OC43 and human coronavirus NL63 infections among hospitalized children $<5$ years of age. Pediatr Infect Dis J. 2014;33(8):814-820.

20. Matoba Y, et al. Detection of the human coronavirus 229E, HKU1, NL63, and OC43 between 2010 and 2013 in Yamagata, Japan. Jpn J Infect Dis. 2015;68(2):138-141.

21. Che XY, et al. Antigenic cross-reactivity between severe acute respiratory syndrome-associated coronavirus and human coronaviruses $229 \mathrm{E}$ and OC43. J Infect Dis. 2005;191(12):2033-2037.

22. Wrapp D, et al. Cryo-EM structure of the 2019nCoV spike in the prefusion conformation. Science. 2020;367(6483):1260-1263.

23. Zhao J, et al. Recovery from the Middle East respiratory syndrome is associated with antibody and T-cell responses. Sci Immunol. 2017;2(14):eaan5393. 